Panorama Económico, Volumen V, No. 10, enero-junio, 2010, pp. 75-96

\title{
MEDIDAS DE POBREZA Y SU EFICIENCIA PARA CAPTAR TRANSFERENCIAS REGRESIVAS DE INGRESO
}

\author{
Genaro Aguilar Gutiérrez*
}

\begin{abstract}
RESUMEN
En épocas de severas depresiones económicas, como la que se vive internacionalmente desde el año 2007, diversas transformaciones que inician en el ámbito económico de las naciones se transfieren hacia su estructura productiva e inevitablemente, hacia los mecanismos que determinan la distribución de la riqueza nacional. En el año 2010 se vive una nueva depresión que viene desde la esfera financiera (con los “Hegde funds” y los derivados financieros detrás de la crisis) de los mercados europeos, pero que traerá repercusiones hacia la distribución de la riqueza dentro de los países. Cuando estas depresiones ocurren también se presentan diversas modificaciones en la estructura social. La pobreza en las naciones más vulnerables aumenta invaria-
\end{abstract}

\footnotetext{
* Doctor en Ciencias Económicas por la Universidade Estadual de Campinas, São Paulo, Brasil. Autor del libro Desigualdad y Pobreza en México, ¿son inevitables?, UNAM, Porrúa e IPN, 2000. Profesor Investigador de la Sección de Estudios de Posgrado e Investigación de la Escuela Superior de Economía del Instituto Politécnico Nacional. Correo electrónico <ggenaro68mx@yahoo.com.br>.
} 
blemente. Para analizar esas modificaciones, que se presentan en los sectores más frágiles de la estructura social, deben utilizarse instrumentos de medición adecuados. Las medidas de pobreza que se emplean en la literatura internacional no responden de igual forma al captar en sus índices las transformaciones sociales que se presentan durante las crisis económicas. Este estudio muestra los cambios que ocurren con las medidas usualmente empleadas en la literatura internacional para cuantificar la pobreza como respuesta a modificaciones en la estructura distributiva del ingreso. Finalmente se concluye que, el índice Sen capta de manera más adecuada lo que ocurre con los más pobres de un país.

Palabras clave: Medida y análisis de la pobreza, ingreso personal, riqueza y su distribución

Clasificación JEL: I32, D31, D63

\begin{abstract}
In times of severe economic depressions like the one we internationally live since 2007, diverse transformations affect the productive structure of nations, and inevitably the mechanisms that determine the distribution of national wealth. In 2010 we are living a new depression that originated in the financial sphere (with "Hedge Funds" and financial derivatives behind the crisis) of European markets, but it will inevitably have repercussions on the distribution of wealth of other countries. Diverse modifications of the social structure arise when these depressions occur. Poverty in the most vulnerable nations invariably increases. Hence, in order to analyze the modifications that arise in the more fragile sectors of the social structure adequate measurement instruments must be used. Poverty measures reported in the international literature don't measure equally, and their indexes fail to adequately capture the social transformations that arise during economic crisis. This study reports on the differences we encounter when trying to quantify poverty as a result of modifications in the distributive income structure using the different measures reported in the international literature. I
\end{abstract}


Medidas de POBREZA Y SU EFICIENCIA PARA CAPTAR

TRANSFERENCIAS REGRESIVAS DE INGRESO

conclude that if we want to adequately measure what happens to the poorest of a country in times of economic depressions we must use the Sen Index.

Keywords: Measure and analysis of poverty, personal income, wealth and its distribution

JEL Classification: I32, D31, D63

\section{INTRODUCCIÓN}

En épocas de estancamiento económico se presentan, generalmente, y mediante diversos mecanismos, transferencias regresivas de ingreso. ${ }^{1}$ Tales transferencias consisten en sustraer una cantidad de ingreso de una persona y agregarlo al de otra persona que anteriormente ya tenía un ingreso igual o mayor que la primera. El principio de Pigou-Dalton establece que, el valor de una medida de desigualdad de la distribución del ingreso debe aumentar cuando se realiza una transferencia regresiva. ${ }^{2}$ ¿Es deseable que una medida de pobreza aumente cuando se presenta una transferencia regresiva?

Diversos autores han analizado lo que sucede con las medidas de desigualdad cuando se presenta una transferencia regresiva de ingreso (ver, por ejemplo, Wolfson,1997; Shorrocks y Foster, 1987; y, Kakwani, 1980). Sin embargo, la variación de las medidas o índices de pobreza ante transferencias regresivas de ingreso, que se presentan a lo largo de la distribución del ingreso de los pobres, no ha sido estudiada sistemáticamente (Hoffmann, 1998; Skorrocks, 1995). La contribución de este artículo consiste en derivar las fórmulas de sensibilidad de las principales medidas de pobreza ante transferencias regresivas de ingreso entre los pobres y comparar las curvas de dicha sensibilidad relativa para una distribución log-normal, semejante a la distribución del ingreso en México.

${ }^{1}$ Un estudio del World Bank (1999) mostró que los mayores incrementos de desigualdad y pobreza se presentaron en México después de las crisis económicas de 1982 y de 1995.

${ }^{2}$ Una revisión de la literatura sobre el principio de Pigou-Dalton se encuentra en Castagnoli y Muliere (1990). 
Además de esta introducción el trabajo contiene tres secciones. En la segunda parte se presentan, de manera suscinta, las principales medidas de pobreza. En la tercera sección se analiza el efecto que tiene una transferencia regresiva entre los pobres sobre el valor de las medidas de pobreza. Se ha dado especial énfasis a la derivación de las fórmulas y a la comparación de las curvas que permiten observar el efecto neto de una transferencia regresiva sobre los índices de Sen y de Foster, Greer y Thorbecke. En la última parte se presentan las conclusiones.

\section{MEDIDAS DE POBREZA}

Dada una población con $n$ personas (o familias), sea $x_{i}$ (con $i=1,2, \ldots, n$ ) el ingreso de la $i$-ésima persona. Considerando que las personas están ordenadas de acuerdo a valores crecientes de ingreso, tenemos:

$$
x_{1} \leq x_{2} \leq \ldots \leq x_{n}
$$

Cuando sólo disponemos de datos sobre el ingreso de las personas (o de las familias), son consideradas pobres todas las personas cuyo ingreso sea igual o inferior a un valor preestablecido, denominado línea de pobreza. Sea $z$ la línea de pobreza, vamos a admitir que hay $p$ personas pobres, ésto es, $x_{p} \leq \mathrm{z} y x_{p+1}>\mathrm{z}$. La proporción de pobres $(H)$ es:

$$
H=\frac{p}{n}
$$

Esta es una medida que capta sólo la extensión o magnitud de la pobreza, siendo insensible a la intensidad de la pobreza. Permite observar qué proporción de pobres existe en una población, pero no nos dice qué tan pobres son. Así, el valor de $H$ no es afectado por la reducción del ingreso de un pobre. 
MEdidAS DE POBREZA Y SU EFICIENCIA PARA CAPTAR

TRANSFERENCIAS REGRESIVAS DE INGRESO

La insuficiencia de ingreso de un pobre es definida como $z-x_{i}(\operatorname{con} i \leq p)$, o sea, es el monto que falta para que su ingreso alcance la línea de pobreza. La insuficiencia de ingreso para todos los pobres en una población es:

$$
\sum_{i=1}^{p}\left(z-x_{i}\right)
$$

Dado un número de personas en situación de pobreza, el valor máximo de esa insuficiencia de ingreso, que sería observado cuando los $p$ pobres tuvieran ingreso nulo, es $p z$.

El cociente entre la insuficiencia de ingreso de los $p$ pobres y su valor máximo es denominado razón de insuficiencia de ingreso $(I)$ :

$$
I=\frac{1}{p z} \sum_{i=1}^{p}\left(z-x_{i}\right)
$$

Sea $m$ el ingreso promedio de los pobres, tenemos:

$$
m=\frac{1}{p} \sum_{i=1}^{p} x_{i}
$$

Sustituyendo (3) en (2), tenemos:

$$
I=1-\frac{m}{Z}
$$

Esta expresión muestra que, dados los valores de z y m, el valor de la razón de insuficiencia de ingreso es insensible al número de pobres.

Las medidas $H$ e $I$ presentan, por lo tanto, defectos y cualidades complementarias. Mientras que $H$ es insensible a la intensidad de la pobreza de cada 
persona (medida por la insuficiencia de ingreso), $I$ es insensible a la magnitud de la pobreza. Una solución es adoptar el producto HI como medida de pobreza.

\subsection{EL ÍNDICE DE SEN}

En un trabajo clásico sobre el tema, Sen (1976) desarrolló una medida que toma en cuenta tanto la magnitud como la intensidad de la pobreza, y también la desigualdad de la distribución del ingreso entre los pobres. Partiendo de un conjunto de axiomas el autor llega a la expresión:

$$
P=\frac{2}{(p+1) n z} \sum_{i=1}^{p}\left(z-x_{i}\right)(p+1-i)
$$

ó

$$
P=\frac{2}{(p+1) n z}\left[\frac{z p(p+1)}{2}-(p+1) \sum_{i=1}^{p} x_{i}+\sum_{i=1}^{p} i x_{i}\right]
$$

ó, también,

$$
P=\frac{p}{n}\left[1-\frac{2 m}{z}+\frac{2}{p(p+1) z} \sum_{i=1}^{p} i x_{i}\right]
$$

En la expresión (5) podemos observar que la insuficiencia de ingreso de cada pobre es ponderada por un número $(p+1-i)$ que indica el orden de la respectiva intensidad de la pobreza. Ese número de orden varía desde 1, para el pobre menos pobre, hasta $p$, para el pobre más pobre.

Se puede verificar que el índice de Sen $(P)$ varía de cero a 1 , con $P=0$ cuando todas las personas tienen ingreso mayor a la línea de pobreza $(z)$ y $P=1$ cuando todas las personas tienen ingreso cero.

Se puede demostrar que el índice de Gini de la distribución del ingreso entre los pobres de nivel $p$ es: 
MEdidAS DE POBREZA Y SU EFICIENCIA PARA CAPTAR

TRANSFERENCIAS REGRESIVAS DE INGRESO

$$
G_{*}=\frac{2}{p^{2} m} \sum_{i=1}^{p} i x_{i}-\left(1+\frac{1}{p}\right)
$$

De las ecuaciones (7) y (8), recordando (1) y (4), y después de una serie de manipulaciones algebraicas, obtenemos:

$$
P=H\left[I+\frac{p}{p+1}(1-I) G_{*}\right]
$$

Como mostró el propio Sen (1976), para $p$ suficientemente grande el índice se reduce a:

$$
P=H\left[I+(1-I) G_{*}\right]
$$

Debe notarse que el índice de pobreza de Sen se reduce al producto $H I$ cuando todos los pobres tienen el mismo ingreso (es decir, la desigualdad de la distribución del ingreso entre ellos es inexistente).

Después del trabajo pionero de Sen fueron presentadas varias propuestas de medidas de pobreza, muchas de ellas consistían en modificaciones al índice de Sen. Una revisión sobre este tema puede ser encontrada en Romão (1982 y 1990).

\subsection{EL ÍNDICE DE FOSTER, GREER Y THORBECKE}

Un nuevo marco en el desarrollo de las medidas de pobreza fue el artículo de Foster, Greer y Thorbecke (1984) en donde se analiza la familia de índices:

$$
\varphi(\alpha)=\frac{1}{n z^{\alpha}} \sum_{i=1}^{p}\left(z-x_{i}\right)^{\alpha}, \text { con } a \geq 0
$$


Esta medida es igual a la proporción de pobres $(H)$ cuando $\alpha=0$, y es igual a $H I$ cuando $\alpha=1$. Se denomina índice de Foster, Greer y Thorbecke al valor obtenido $\operatorname{con} \alpha=2$ :

$$
\varphi(\alpha)=\frac{1}{n z^{2}} \sum_{i=1}^{p}\left(z-x_{i}\right)^{2}
$$

Puede demostrarse que $\varphi(\alpha)$, de la misma manera que el índice de Sen varía de cero a 1 , con $\varphi(\alpha)=0$ cuando todas las personas tienen ingreso mayor que z y $\varphi(\alpha)=1$ cuando todos los ingresos son iguales a cero.

El coeficiente de variación de los ingresos de las $p$ personas es:

$$
C_{*}=\frac{1}{m}\left(\frac{1}{p} \sum_{i=1}^{p} x_{i}^{2}-m^{2}\right)^{1 / 2}
$$

Entonces,

$$
C_{*}^{2}=\frac{1}{p m^{2}} \sum_{i=1}^{p} x_{i}^{2}-1
$$

De las ecuaciones (12) y (13), después de algunas transformaciones algebraicas, obtenemos:

$$
\varphi=H\left[I^{2}+(1-I)^{2} C_{*}^{2}\right]
$$

Existe cierta analogía entre las expresiones (10) y (14). Tanto el índice de Sen como el de Foster, Greer y Thorbecke son funciones de la proporción de pobres $(H)$, de la razón de insuficiencia de ingreso $(I)$ y de una medida de desigualdad de la distribución del ingreso entre las personas pobres.

Si no existiera desigualdad entre los pobres, tenemos que $C_{*}=0$ y $\varphi=H I^{2}$. 
MEDIDAS DE POBREZA Y SU EFICIENCIA PARA CAPTAR

TRANSFERENCIAS REGRESIVAS DE INGRESO

Una propiedad importante de la familia de índices (11) ampliamente documentada es su descomposición, cuando una población con $N$ personas es dividida en $k$ grupos o regiones. Vamos a indicar el ingreso de la $i$-ésima persona del $h$-ésimo grupo por $x_{h i}$, con $i=1,2, \ldots, n_{h}$ y $h=1,2, \ldots, k$. La participación del $h$-ésimo grupo en la población es $\pi_{h}=n_{h} / N$. Vamos a admitir que la línea de pobreza $(z)$ es la misma para todos los grupos y que, dentro de cada grupo, los ingresos estan ordenados de manera que:

$$
x_{h 1} \leq x_{h 2} \leq \ldots \leq x_{h p h} \leq z \leq \ldots \leq x_{h n h}
$$

Entonces, $p_{h}$ es el número de pobres del $h$-ésimo grupo y el índice (11) dentro de ese grupo es:

$$
\varphi_{h}(\alpha)=\frac{1}{n_{h} z^{\alpha}} \sum_{i=1}^{p_{h}}\left(z-x_{h i}\right)^{\alpha}
$$

El índice para toda la población es:

$$
\varphi(\alpha)=\frac{1}{N z^{\alpha}} \sum_{h=1}^{k} \sum_{i=1}^{p_{h}}\left(z-x_{h i}\right)^{\alpha}
$$

De las expresiones (15) y (16) se deriva fácilmente que:

$$
\varphi(\alpha)=\sum_{h=1}^{k} \pi_{h} \varphi_{h}(\alpha)
$$

Ese resultado muestra que, el índice $\varphi(\alpha)$ para toda la población es igual a la suma de los valores del producto del índice dentro de cada grupo por la respectiva participación en la población. El producto $\pi_{h} \varphi_{h}(\alpha)$ corresponde, por lo tanto, a la contribución del $h$-ésimo grupo a la pobreza global. 
Si la división de la población fuera hecha conforme a las regiones de un país y la línea de pobreza fuera diferente en cada región, la expresión (15) puede ser adaptada sustituyendo $z$ por la línea de pobreza específica de la $h$-ésima región $\left(z_{h}\right)$, y la expresión (17) pasaría a ser, por definición, la medida de pobreza global.

La expresión (17) muestra que, fijada la distribución de la población por los $k$ grupos, alteraciones en el ingreso de las personas que causen un aumento del índice $\varphi_{h}(\alpha)$ dentro de uno o más grupos llevarán, necesariamente, a un aumento de la medida de pobreza en toda la población.

Cabe resaltar que el índice de pobreza de Sen, debido a su asociación con el índice de Gini, no presenta las propiedades de descomposición de la familia de índices de Foster, Greer y Thorbecke. Inclusive pueden construirse ejemplos de una población dividida en grupos en que determinadas alteraciones en los ingresos causan aumento del índice de Sen dentro de todos ellos y, al mismo tiempo, disminución del índice de Sen para toda la población.

\section{EFECTO DE LA REDUCCIÓN DEL INGRESO DE UN POBRE SOBRE LAS MEDIDAS DE POBREZA}

Las mutaciones que sufre la distribución del ingreso en un país son diversas dependiendo, fundamentalmente, de la estructura económica de dicha nación. En general, en épocas de crisis el efecto neto de las transferencias de ingreso empeora la situación relativa de los individuos en situación de pobreza. No obstante, si el cambio de una parte del ingreso de los pobres hacia los no tan pobres se presenta simultáneamente a una transferencia de ingreso de otro segmento de los no tan pobres hacia otros más pobres, el efecto compensador que implican estos movimientos puede resultar en indicadores de pobreza que permanecen sin alteración alguna. ¿Qué medida de pobreza puede captar mejor estos movimientos? ¿El índice de Sen es suficientemente bondadoso como para mostrar lo que está pasando con los pobres en épocas de crisis? Estas preguntas serán respondidas en esta parte del artículo. 
Así, en esta sección veremos cómo la reducción del ingreso de una persona pobre afecta las medidas de pobreza, mostrando que ese efecto varía de acuerdo al ingreso inicial de dicha persona pobre. ${ }^{3}$

Sea $\theta$ el valor que es sustraído del ingreso $x_{h}$ de una persona pobre $(h \leq p)$. El efecto de esta reducción de ingreso de una persona pobre sobre el valor del índice de pobreza será, en general, función de $\theta$. Para facilitar las comparaciones vamos a considerar el valor del límite:

$$
\lim _{\theta \rightarrow 0} \frac{\Delta \pi}{\theta}=\lim _{\theta \rightarrow 0} \frac{\partial \pi}{\theta}
$$

donde $\pi$ representa un índice de pobreza cualquiera.

De acuerdo con la ecuación (11), después de la reducción del ingreso de una persona pobre $\left(x_{h}\right)$ a un nivel inferior (tal como $x_{h}-\theta$ ) el índice $\varphi_{h}(\alpha)$ queda como:

$$
\varphi(\alpha)=\frac{1}{n z^{\alpha}}\left[\sum_{i \neq h}\left(z-x_{i}\right)^{\alpha}+\left(z-x_{h}+\theta\right)^{\alpha}\right]
$$

Entonces,

$$
\frac{\partial \varphi(\alpha)}{\partial \theta}=\frac{\alpha\left(z-x_{h}+\theta\right)^{\alpha-1}}{n z^{\alpha}}
$$

$\mathrm{y}$

${ }^{3}$ Para la familia de índices propuesta por Foster, Greer y Thorbecke, este análisis de la sensibilidad a la reducción del ingreso de un pobre está implícito en la discusión que los autores hacen sobre la obediencia de los índices a los axiomas de monotonicidad, transferencia y sensibilidad a transferencias. 


$$
\begin{aligned}
& \text { Genaro Aguilar Gutiérrez } \\
& \lim _{\theta \rightarrow 0} \frac{\Delta \varphi(\alpha)}{\theta}=\frac{\alpha\left(z-x_{h}\right)^{\alpha-1}}{n z^{\alpha}}
\end{aligned}
$$

Sabemos que, para $\alpha=0$ el índice $\varphi_{h}(\alpha)$ es la proporción de pobres. La expresión (19) es igual a cero cuando $\alpha=0$, confirmando el hecho de que la proporción de pobres es insensible a la reducción del ingreso de un pobre.

Cuando $\alpha=1$ el índice $\varphi(\alpha)$ es igual a HI. De acuerdo con la ecuación (19), con $\alpha=1$ tenemos:

$$
\lim _{\theta \rightarrow 0} \frac{\Delta(H I)}{\theta}=\frac{1}{n z},
$$

mostrando que la sensibilidad del índice de Foster, Greer y Thorbecke es directamente proporcional a la insuficiencia de ingreso de la persona pobre cuyo ingreso ha sido reducido.

Para $\alpha=2$ tenemos, de acuerdo con (19):

$$
\lim _{\theta \rightarrow 0} \frac{\Delta \varphi}{\theta}=\frac{2\left(z-x_{h}\right)}{n z^{2}}
$$

Expresión que muestra que la sensibilidad del índice de Foster, Greer y Thorbecke es directamente proporcional a la insuficiencia de ingreso de la persona pobre cuyo ingreso se ha visto reducido.

Para obtener la expresión de la sensibilidad del índice de Sen a una reducción de ingreso $\left(x_{h}\right.$ con $\left.h \leq p\right)$ del $h$-ésimo pobre, vamos a admitir que la persona cuyo ingreso será reducido $\left(x_{h}-\theta\right)$ pasa a ocupar la $g$-ésima posición (con $g<h$ ). Con ello, las personas con ingreso $x_{g}, x_{g+1}, \ldots, x_{h-1}$ pasarán a ocupar posiciones de orden una unidad arriba de sus posiciones anteriores. De acuerdo con la ecuación (6), la consecuente alteración en el valor del índice de Sen es: 


$$
\begin{aligned}
& \text { Medidas de pobreza y su eficiencia para Captar } \\
& \text { TRANSFERENCIAS REGRESIVAS DE INGRESO } \\
& \Delta P=\frac{2}{(p+1) n z}\left[\theta(p+1)+g\left(x_{h}-\theta\right)-h x_{h}+\sum_{i=g}^{h-1}(i+1) x_{i}-\sum_{i=g}^{h-1} i x_{i}\right]
\end{aligned}
$$

ó

$$
\Delta P=\frac{2}{(p+1) n z}\left[\theta(p+1-g)-x_{h}(h-g)+\sum_{i=g}^{h-1} x_{i}\right]
$$

ó, también,

$$
\Delta P=\frac{2}{(p+1) n z}\left[\theta(p+1-g)-\sum_{i=g}^{h-1}\left(x_{h}-x_{i}\right)\right]
$$

En el caso de una distribución discreta, si $\theta$ fuera muy pequeña no habría reordenación de las personas y tendríamos $g=h$. Entonces:

$$
\lim _{\theta \rightarrow 0} \frac{\Delta P}{\theta}=\frac{2(p+1-h)}{(p+1) n z}
$$

En el caso de una distribución continua, con función de densidad $f(x)$ y una función de distribución $F(x)$, la expresión (22) corresponde a:

$$
\Delta P=\frac{2}{n z F(z)}\left\{\theta\left[F(z)-F\left(x_{h}-\theta\right)\right]-\int_{x_{h-\theta}}^{x_{h}}\left(x_{h}-x\right) \partial F(x)\right\}
$$

\section{Como}

$$
\lim _{\theta \rightarrow 0} \frac{1}{\theta} \int_{x_{h-\theta}}^{x_{h}}\left(x_{h}-x\right) \partial F(x)=0
$$


Concluimos que:

$$
\lim _{\theta \rightarrow 0} \frac{\Delta P}{\theta}=\frac{2\left[F(z)-F\left(x_{h}\right)\right]}{n z F(z)}
$$

Si el ingreso estuviera uniformemente distribuido en el intervalo de $a$ hasta $b$, tenemos $F(x)=(x-a) /(b-a)$ y, como $a<z<b$, podemos verificar que:

$$
\lim _{\theta \rightarrow 0} \frac{\Delta P}{\theta}=\frac{2\left(z-x_{h}\right)}{n z(z-a)}
$$

Recordando la expresión (21) observamos que, en este caso, si $a=0$ la sensibilidad del índice de Sen es idéntica a la sensibilidad del índice de Foster, Greer y Thorbecke.

Para visualizar y comparar la sensibilidad de las diversas medidas de pobreza $(\pi)$ a la reducción $(\theta)$ en el ingreso $\left(x_{h}\right)$ de una persona pobre, vamos a construir curvas que muestran el valor incrementado de dichas medidas; cuando el valor transferido de ingreso $(\theta)$ de una persona a otra tiende a cero:

$$
\lim _{\theta \rightarrow 0} \frac{\Delta \pi}{\theta}
$$

Esto equivale a observar lo que pasa con cualquier medida de pobreza cuando se presenta una transferencia infinitesimal de ingreso desde una persona pobre hacia una persona no tan pobre.

Veremos que dicho valor varía en función del ingreso inicial de la persona pobre $\left(x_{h}\right)$. Para hacer esto, en el caso del índice de Sen, es necesario conocer la forma de la distribución del ingreso. Vamos a considerar aquí una distribución log-normal. Cualquier distribución empírica del ingreso sigue un comportamiento aproximadamente log-normal, con asimetría positiva. Con- 
Medidas de Pobreza y SU EFICIENCIA PARA CAPTAR

TRANSFERENCIAS REGRESIVAS DE INGRESO

sideraremos que el promedio y la varianza de los logaritmos de los ingresos son, respectivamente, $\mu=-0.7$ y $\sigma^{2}=1.5$. En este caso el ingreso promedio es 1.05 ; la mediana del ingreso es 0.50 ; la moda es 0.11 y el índice de Gini es 0.61 . Se trata de las características que tiene la distribución del ingreso familiar per cápita en México en $2008,{ }^{4}$ y tomamos como unidad de medida el mayor salario mínimo vigente en el país para el mismo año.

Para esa distribución log-normal, adoptando una línea de pobreza $z=0.25$ (salarios mínimos per cápita), obtenemos $H=0.2876$; ingreso promedio de los pobres $(m)$ igual a $0.1357 ; H I=0.1315 ; P=0.1740 ; \varphi(\alpha=1.5)=0.09990$; $\varphi(\alpha=2.0)=0.07898$ у $\varphi(\alpha=2.5)=0.06425$.

Considerando la misma distribución log-normal, pero adoptando una línea de pobreza dos veces mayor $(z=0.5$, es decir medio salario mínimo por persona), obtenemos $H=0.5022 ; m=0.2332 ; H I=0.2680 ; P=0.3438$; $\varphi(\alpha=1.5)=0.2148 ; \varphi(\alpha=2.0)=0.1777$ у $\varphi(\alpha=2.5)=0.1504$.

Las curvas que muestran cómo la sensibilidad definida por la ecuación (25) varía en función de $x_{h}$, con $z=0.25 \mathrm{y} z=0.5$, se presentan en las gráficas 1 y 2 , respectivamente. De acuerdo con la expresión (20), la sensibilidad de $H I=\varphi(\alpha=1.0)$ es representada por una recta horizontal con ordenada $1 /(n z)$, que es igual a 0.004 , para $z=0.25$; y es igual a 0.002 , para $z=0.5$. De acuerdo con (21), la sensibilidad del índice de Foster, Greer y Thorbecke es ilustrada por una recta decreciente; cuando $x_{h}=0$ el efecto es $2 /(n z)$, que es igual a 0.008 para $z=0.25$; y, es igual a 0.004 para $z=0.5$. Las sensibilidades de $\varphi(\alpha=1.5)$ у $\varphi(\alpha=2.5)$ son representadas por curvas que son, respectivamente, cóncava y convexa.

Cuando la insuficiencia de ingreso es pequeña la curva que muestra la sensibilidad del índice de Sen es convexa. En la medida en que aumenta la insuficiencia de ingreso su inclinación aumenta, en términos absolu-

\footnotetext{
${ }^{4}$ Los cálculos fueron realizados con los microdatos más recientes de la Encuesta Nacional de Ingreso y Gasto de los Hogares de México, que corresponden al año de 2008.
} 
tos, alcanzando un máximo en el punto correspondiente a la moda de la distribución del ingreso (que en este caso es $x=0.11$ ). Para ingresos $\left(x_{h}\right)$ abajo de la moda, la curva de sensibilidad del índice de Sen es cóncava. En el límite, para $x_{h}=0$, la inclinación de la curva de sensibilidad es igual a cero y el efecto de la reducción del ingreso de una persona pobre sobre el índice de Sen es igual a su efecto sobre el índice de Foster, Greer y Thorbecke.

La posición de la curva de sensibilidad del índice de Sen, en relación a las curvas referentes a los índices $\varphi(\alpha)$, depende de la línea de pobreza adoptada. Podemos ver que, como se muestra en la Gráfica 1 , cuando z $=0.25$ la curva de sensibilidad del índice de Sen se confunde con aquella referente al índice $\varphi(\alpha=1)$ para valores bastante pequeños de insuficiencia de ingreso. Si $z=0.5$ la curva de sensibilidad del índice de Sen permanece muy próxima de aquella referente al índice $\varphi(\alpha=2.3)$ en el intervalo $0.35<x_{h}<0.5$ (que no aparece en la Gráfica 2).

Tomando en cuenta la semejanza de las curvas de sensibilidad del índice de Sen $(P)$ y del índice de Foster, Greer y Thorbecke $(\varphi)$, y considerando las propiedades de descomposición de $\varphi$ y el hecho de que su cálculo es más simple, es probable que el uso de $\varphi$ predomine, cada vez más, sobre el uso de $P$.

Vamos a considerar una transferencia regresiva de ingreso entre dos personas pobres de manera que el ingreso del pobre beneficiado por la transferencia no supere la línea de pobreza. Sean $x_{h}$ y $x_{h}+\mathrm{w}($ con $\mathrm{w}>0)$ los ingresos antes de la transferencia regresiva. Después de la transferencia (de un monto $\theta$ ) los ingresos pasan a ser $x_{h}-\theta$ y $x_{h}+w+\theta$, admitiendo que $x_{h}+w+$ $\theta \leq z$. Vamos a fijar los valores de w y $\theta$ y a analizar cómo el efecto de la transferencia regresiva sobre la medida de pobreza depende de $x_{h}$ (el ingreso inicial del pobre afectado). El efecto de tal transferencia regresiva está asociado a la forma de la curva de sensibilidad de esa medida de pobreza de la reducción del ingreso de un pobre. Para una curva de sensibilidad horizontal (que es el caso de $H I$ ), la transferencia regresiva no afecta el valor de la medida de pobreza, pues el efecto de la reducción del ingreso $x_{h}$ es exactamente compensado por el efecto del aumento del ingreso $x_{h}+$ w. En el caso del índice de Foster, Greer y Thorbecke, cuya sensibilidad es una recta de- 
MEdidas de POBREZA Y SU EFICIENCIA PARA CAPTAR

TRANSFERENCIAS REGRESIVAS DE INGRESO

\section{Gráfica 1}

Sensibilidad de diversas medidas de pobreza en función del ingreso de un pobre y ante su reducción,

para una línea de pobreza $\mathrm{z}=\mathbf{0 . 2 5}$

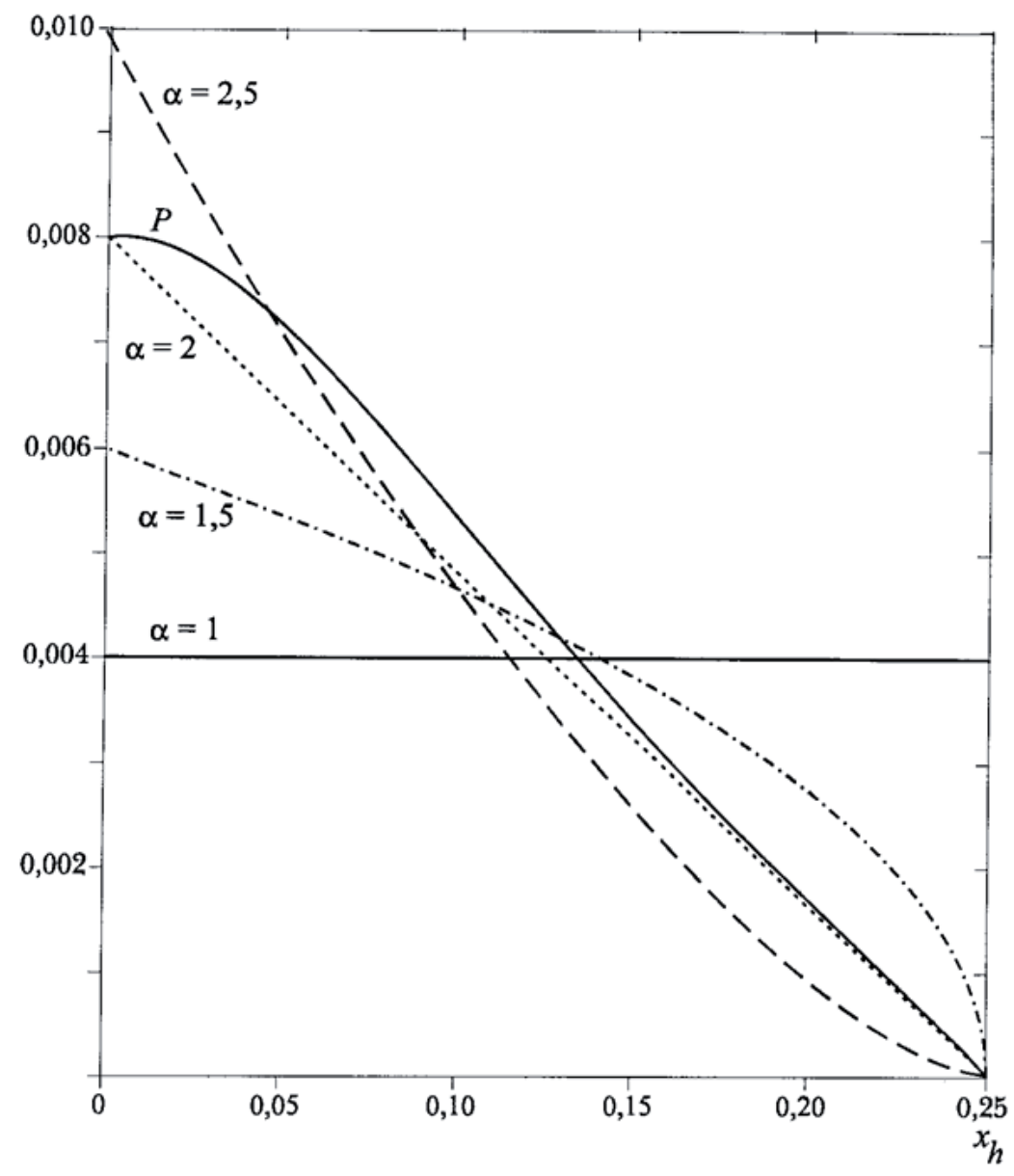

Fuente: Elaboración propia. 
Genaro Aguilar Gutiérrez

\section{GráficA 2}

Sensibilidad de diversas medidas de pobreza en función del ingreso de un pobre y ante su reducción, para una línea de pobreza $z=0.5$

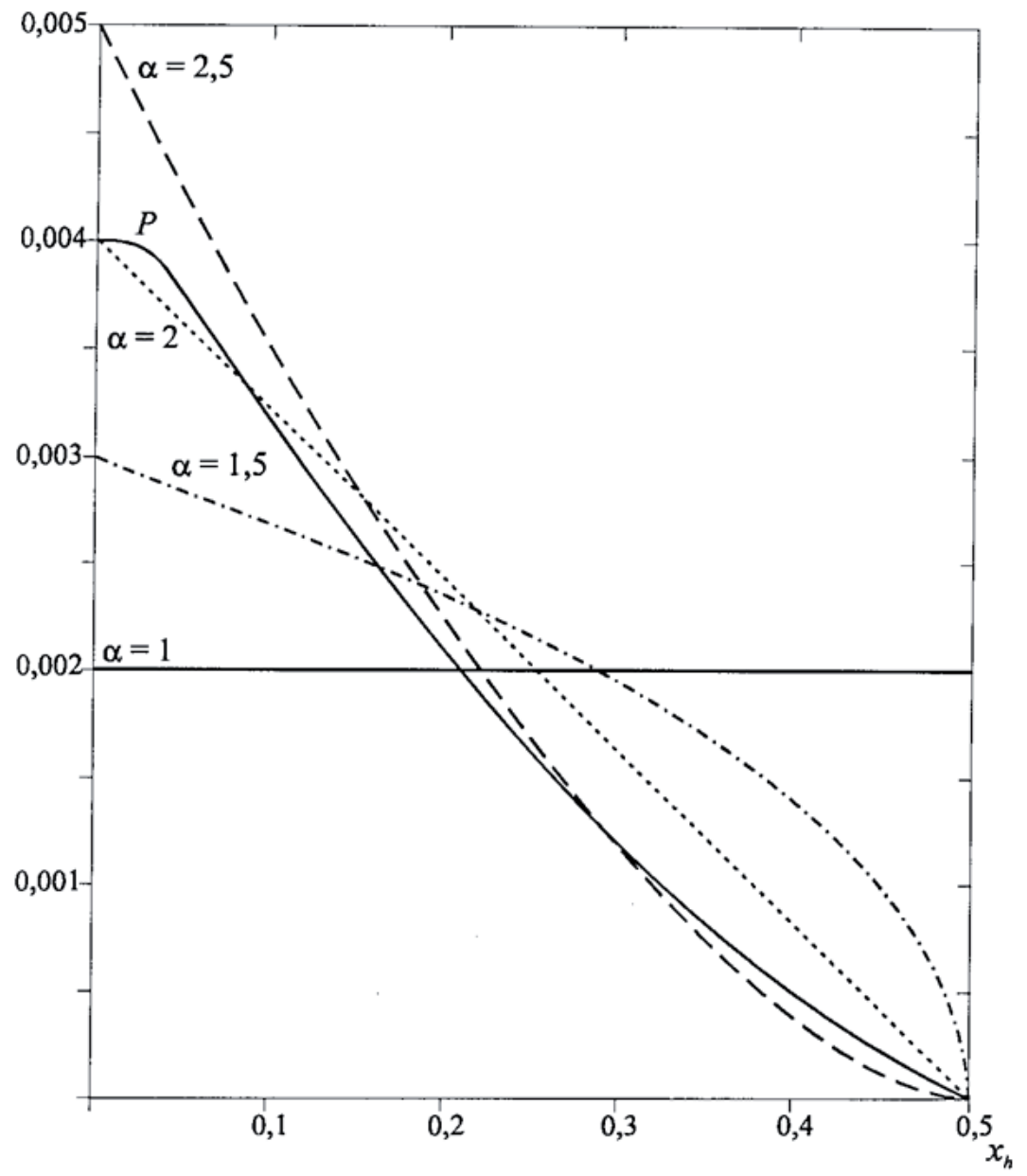

Fuente: Elaboración propia. 
Medidas de pobreza y SU EFICIENCIA PARA CAPTAR

TRANSFERENCIAS REGRESIVAS DE INGRESO

creciente, el efecto de la transferencia regresiva no depende del ingreso inicial del pobre afectado, $x_{h}$. Si la curva de sensibilidad es convexa, el efecto de la transferencia regresiva disminuye con el ingreso $x_{h}$ (aumenta con la insuficiencia de ingreso). Finalmente, si la curva de sensibilidad ante una reducción del ingreso de una persona pobre es cóncava entonces, el efecto de la transferencia regresiva crece con el ingreso $x_{h}$ (disminuye cuando aumenta la insuficiencia de ingreso).

Se prueba, por lo tanto, que para los índices $\varphi(\alpha)$ el efecto de la transferencia regresiva sólo aumenta con el nivel de insuficiencia de ingreso, si $\alpha>2$ (hecho ya establecido por Foster, Greer y Thorbecke, 1984, p.763). En el caso del índice de Sen, el efecto de la transferencia regresiva sólo crece con la insuficiencia de ingreso para ingresos superiores a la moda de la distribución.

El hecho de que el efecto de la transferencia regresiva sobre el índice de Sen aumente con el ingreso $x_{h}$, mientras que sea menor a la moda, fue señalado por Kakwani (1980), que lo consideró una limitación seria de esa medida de pobreza. Como el autor admite, la línea de pobreza es menor que la moda de la distribución y concluye que "[...] Sen`s measure gives the least weigth to a transfer from the worst off poor and the weight increases with the level of income" (p. 443).

En contraposición a la crítica de Kakwani al índice de Sen, cabe discutir tres cuestiones.

1. El supuesto de que la línea de pobreza está siempre por debajo de la moda es razonable para países desarrollados, pero en los estudios sobre pobreza en México han sido adoptadas, en general, líneas de pobreza superiores al ingreso modal. Para una distribución log-normal con un índice igual o superior a 0.58 , la proporción de personas con ingreso por debajo de la moda llega a estar entre 10 y 13\%, mucho menos de lo que las estimaciones usuales registran al medir la pobreza en el país. ${ }^{5}$

\footnotetext{
${ }^{5}$ Incluso las estimaciones oficiales de la Secretaría de Desarrollo Social hablan de más de 25\% de personas en situación de pobreza.
} 
2. Es recomendable que un indicador de pobreza adecuado presente sensibilidad a la reducción del ingreso de un pobre, creciente con el valor de la insuficiencia de ingreso, condición que es satisfecha por el índice de Sen y por los índices $\varphi(\alpha)$, con $\alpha>1$. Exigir que el efecto de una transferencia regresiva aumente con la insuficiencia de ingreso corresponde a exigir que la curva de sensibilidad a la reducción del ingreso de un pobre sea convexa. ¿Sería esa una cualidad necesaria de una buena medida de pobreza? ¿No se estaría "transfiriendo" para las medidas de pobreza las cualidades deseables de las medidas de desigualdad? Debemos recordar que la propia noción de desigualdad está directamente asociada al efecto de una transferencia regresiva de ingreso en la condición Pigou-Dalton.

3. El hecho de que la sensibilidad del índice de Sen a la reducción del ingreso de un pobre crezca cada vez más lentamente con la insuficiencia de ingreso cuando el ingreso se aproxima a cero, puede incluso ser considerada una cualidad deseable, en la medida en que tales ingresos no corresponden al ingreso real de las personas, como es el caso, por ejemplo, de las personas ocupadas con ingreso declarado igual a cero y que son miembros no remunerados de las familias de pequeños agricultores.

El principal defecto del índice de Sen es, indudablemente, la dificultad de usarlo en análisis que involucran la descomposición de la población en grupos.

\section{CONCLUSIONES}

El conocimiento de la sensibilidad relativa de las diversas medidas de pobreza ante transferencias regresivas de ingreso entre los pobres, efectuadas en varios puntos de la distribución, es fundamental para la selección de las medidas apropiadas conforme a los objetivos de la investigación que se realiza. Particularmente lo es cuando se analiza lo que sucede en épocas de crisis, en que tales transferencias son comunes. 
Medidas DE POBREZA Y SU EFICIENCIA PARA CAPTAR

TRANSFERENCIAS REGRESIVAS DE INGRESO

En este trabajo proponemos comparar el efecto de transferencias regresivas de ingreso entre los pobres de las medidas usuales en la literatura internacional que cuantifica la pobreza. El índice de Sen es muy sensible a transferencias de ingreso que se realizan entre las personas muy pobres y lo capta de mejor manera que el índice de Foster, Greer y Thorbecke.

La obediencia de la condición Pigou-Dalton es esencial para medidas de desigualdad pero, dadas las consideraciones en torno al índice de Sen mostramos que, tratándose de medidas de pobreza dicha condición no necesariamente debe cumplirse. La bondad del índice de Sen no reside en ello, sino en la capacidad de mostrar movimientos de ingreso entre los pobres que, de otra manera, se ocultarían en un análisis riguroso de la evolución de la pobreza.

El investigador que busque una medida de pobreza medianamente sensible a transferencias de ingreso entre los muy pobres, pero que requiera un análisis

de descomposición de la pobreza, deberá usar el índice de Foster, Greer y Thorbecke.

En última instancia se debe tomar en cuenta que, un análisis de pobreza requiere la combinación inteligente de las herramientas que proporciona la Teoría de la Pobreza.

\section{BIBLIOGRAFÍA}

Castagnoli, E., P. Muliere, “A note on inequality measures and the Pigou-Dalton principle of transfers”, en C. Dagun, M. Senga, orgs., Income and wealth distribution, inequality and poverty, Proceedings, 1989, Springer-Verlag, Berlin, 1990.

Foster, J., J. Greer and E. Thorbecke, “A Class of Decomposable Poverty Measures”, Econometrica, vol. 52, n. 3, p. 761-766, 1984.

Hoffmann, Rodolfo, Distribuição de Renda. Medidas de Desigualdade e Pobreza, Editora da Universidade de São Paulo, 1998.

Romão, M. E. C., "Índices de Pobreza: Alternativas, Decomposição e Uso com Dados Agregados”, Estudos Econômicos, vol.12, n.3; p. 51-65; Brasil, 1982. 
“Pobreza: Conceito e Mensuração”, Relatório de Pesquisa, Recife, Pimes-UFPE, Brasil, 1990.

Sen, A., "Poverty: an Ordinal Approach to Measurement", Econometrica, vol. 44, n.2, p. 219-231, 1976

Shorrocks, Anthony, F., "Revisiting the Sen Poverty Index”, Econometrica, vol. 63, n.5, p.1225-1230, september 1995.

Shorrocks, A. F., and J. E. Foster, “Transfer sensitive inequality measures”, Review of Economic Studies, vol. 54, pp. 485-497, 1987.

Wolfson, Michael, C., "Divergent Inequalities: Theory and Empirical Results”, Review of Income and Wealth, Series 43, number 4, pp. 401-421, december 1997.

World Bank., "Earnings Inequality after Mexico's Economic and Educational Reforms”, Main Document Volume 1. Latin America and the Caribbean Region Poverty Reduction and Economic Management Division, september 1999. 